\title{
Declining Intensive Care Unit Mortality of COVID-19: A Multi-Center Study
}

\author{
Sohaib Roomi ${ }^{\mathrm{a}, \mathrm{g}}$, Syed Omar Shah ${ }^{\mathrm{b}}$, Waqas Ullah ${ }^{\mathrm{a}}$, Shan Ul Abedinª, Karyn Butlerc, \\ Kelly Schiers ${ }^{\mathrm{d}}$, Benjamin Kohle, Erika Yoo ${ }^{\mathrm{f}}$, Matthew Vibbert ${ }^{\mathrm{b}}$, Jack Jallo ${ }^{\mathrm{b}}$
}

\begin{abstract}
Background: Coronavirus disease 2019 (COVID-19) mortality has waned significantly over time; however, factors contributing towards this reduction largely remain unidentified. The purpose of this study was to evaluate the trend in mortality at our large tertiary academic health system and factors contributing to this trend.

Methods: This is a retrospective cohort study of intensive care unit (ICU) patients diagnosed with COVID-19 between March and August 2020 admitted across 14 hospitals in the Philadelphia area. Collected data included demographics, comorbidities, admission risk of mortality score, laboratory values, medical interventions, survival outcomes, hospital and ICU length of stay (LOS) and discharge disposition. Chi-square $\left(\chi^{2}\right)$ test, Fisher exact test, Cochran-MantelHaenszel method, multinomial logistic regression models, independent sample $t$-test, Mann-Whitney U test and one-way analysis of variance (ANOVA) were used.
\end{abstract}

Results: A total of 1,204 patients were included. Overall mortality was $39 \%$. Mortality declined significantly from $46 \%$ in March to $14 \%$ in August 2020 ( $\mathrm{P}<0.05)$. The most common underlying comorbidities were hypertension $(60.2 \%)$, diabetes mellitus (44.7\%), dyslipidemia $(31.6 \%)$ and congestive heart failure (14.7\%). Hydroxychloroquine (HCQ) use was more commonly associated with the patients who died, while the use of remdesivir, tocilizumab, steroids and duration of these medications were not significantly different. Peak values

Manuscript submitted February 16, 2021, accepted February 23, 2021

Published online March 19, 2021

aDepartment of Medicine, Abington Hospital, Jefferson Health, Philadelphia, PA, USA

${ }^{b}$ Department of Neurological Surgery, Vickie and Jack Farber Institute for Neuroscience, Thomas Jefferson University, Philadelphia, PA, USA

${ }^{\mathrm{c}}$ Department of Surgery, Division of Surgical Critical Care, Abington Hospital, Jefferson Health, Philadelphia, PA, USA

${ }^{\mathrm{d}}$ Department of Medicine, Jefferson Health New Jersey, Washington Township, NJ, USA

eDepartment of Anesthesiology, Aria-Jefferson Health, Philadelphia, PA, USA fDepartment of Medicine, Thomas Jefferson University, Philadelphia, PA, USA

'Corresponding Author: Sohaib Roomi, Department of Medicine, Abington Hospital, Jefferson Health, 1200 Old York Road, Abington, PA 19001, USA. Email: roomigujjar251@gmail.com

doi: https://doi.org/10.14740/jocmr4452 of ferritin, lactate dehydrogenase (LDH), C-reactive protein (CRP) and $\mathrm{D}$-dimer levels were significantly higher in patients who died $(\mathrm{P}<$ $0.05)$. The mean hospital LOS was significantly longer in the patients who survived compared to the patients who died (18 vs. $12, \mathrm{P}<0.05)$.

Conclusions: The mortality of patients admitted to our ICU system significantly decreased over time. Factors that may have contributed to this may be the result of a better understanding of COVID-19 pathophysiology and treatments. Further research is needed to elucidate the factors contributing to a reduction in the mortality rate for this patient population.

Keywords: COVID-19; SARS-CoV-2; Mortality; Intensive care unit

\section{Introduction}

Coronavirus disease 2019 (COVID-19) caused by severe acute respiratory syndrome coronavirus 2 (SARS-CoV-2) spread across the globe in a matter of months. On March 11, 2020, the World Health Organization (WHO) labeled it as a pandemic and issued emergency use authorization (EUA) for various drugs to counter the devastation caused by this "once in a century pandemic" [1]. Various off-label and compassionate use drugs have been trialed based on in vitro activity against SARS-CoV-2. Efficacy has been limited except for low-dose dexamethasone and remdesivir as per preliminary data of the Recovery trial $[2,3]$. By the end of August 2020, more than 30 million cases have been reported worldwide, 6.5 million cases in the USA and a total death toll of around 200,000 in the USA.

Although the total number of cases is increasing over time, recent data from Europe suggest that the mortality rate from COVID-19 has declined in all age groups, and the older age groups drive the overall reduction. In Germany, the case fatality rate declined from $29 \%$ in mid-April to $11 \%$ by the end of July in patients aged 80 and above, while a sharper decline from $9 \%$ in March to $2 \%$ in July was observed in the age group between 60 and 79 years [4]. However, the intensive care unit (ICU) mortality rate varies significantly between countries and over time, which renders it to be highly uncertain. A recent study from Emory University reported an ICU mortality rate of $30.9 \%$ at the end of April 2020, which was significantly lower compared to earlier mortality rates of $50-70 \%$ as per previous reports from China, Italy and Seattle [5]. 
The primary outcome of this study was to evaluate the trend in COVID-19 mortality at our large academic health system consisting of 14 hospitals serving the greater Philadelphia area and southern New Jersey. Secondary outcomes evaluated differences in demographics, comorbidities, ICU interventions and outcomes of the patients who survived compared to patients who died during the study period.

\section{Materials and Methods}

\section{Study design and participants}

This is a retrospective cohort study of all patients with confirmed COVID-19 infection who were admitted to the ICUs across 14 affiliated hospitals of the Jefferson Health System between March 1, 2020, and August 31, 2020. Jefferson Health is a multi-state non-profit health system with its main hospital located in Center City, Philadelphia. With its affiliate hospitals, the system serves the population of the greater Philadelphia area and southern New Jersey. During the study period, all patients admitted to the ICU were cared for by a dedicated critical care team with standard ICU staffing ratios. There was no shortage of medications or critical care equipment during the study period. The Institutional Review Board approved this study and the Research Ethics Committee waived the requirement for informed consent.

\section{Data collection}

Data were extracted from electronic medical records (Epic, Verona WI and Sunrise, Chicago, IL) using a standardized data collection form by our data analytics team. Collected data included patient demographics (age, gender, ethnicity), comorbidities (diabetes mellitus, hypertension, dyslipidemia, heart failure, smoking, chronic obstructive pulmonary disease, asthma, atrial fibrillation, chronic kidney disease, and stroke), medications (hydroxychloroquine (HCQ), tocilizumab (TCZ), remdesivir, steroids), respiratory support (mechanical intervention, bilevel positive airway pressure (BiPAP), high-flow nasal cannula (HFNC)), and adjunctive interventions (extracorporeal membrane oxygenation (ECMO), epoprostenol sodium, nitric oxide). Laboratory values (C-reactive protein (CRP), erythrocyte sedimentation rate (ESR), D-dimer, ferritin, fibrinogen, lactate dehydrogenase (LDH) were collected on admission, day 1, day 10 and peak values during the hospital stay (highest lab values irrespective of the day of hospitalization). Admission risk of mortality score was determined using software developed by Vizient Inc. (Irving, Texas) and was classified as minor, moderate, major, and extreme. Patient outcomes and length of stay (LOS) were also collected. Realtime polymerase chain reaction (RT-qPCR) was used to detect the virus in nasopharyngeal, tracheal, or other respiratory specimens; and patients were considered to have confirmed infection if the initial or repeat test was positive. For patients with readmission during the study period, data from the first admission were used.

\section{Statistical analysis}

A Chi-square $\left(\chi^{2}\right)$ test was used for comparison of categorical data and Fisher exact test was adopted if the expected count in more than $20 \%$ cells was less than 5 . Continuous variables were presented as means and standard deviations, while categorical variables were reported in percentages and proportions. To quantify the association between the dichotomous categorical variables, an unadjusted odds ratio (uOR) was obtained using a Cochran-Mantel-Haenszel method. Multinomial regression analysis was used to adjust our outcome for all variables collected. For normally and abnormally distributed continuous data, an independent sample $t$-test and Mann-Whitney $\mathrm{U}$ test were used, respectively. A one-way analysis of variance (ANOVA) was used to compare differences in the mean of continuous variables for multiple in-hospital complications. A two-sided $\alpha<0.05$ was considered statistically significant with corroborating inference from a $95 \%$ confidence interval (CI). Statistical analyses were performed using the SPSS software (version 25, IBM Corp).

\section{Results}

\section{Demographics and baseline characteristics}

A total of 1,204 patients were admitted to the ICU with confirmed SARS-CoV-2 infection. There were 715 males $(59.3 \%)$, and a majority of patients were white $(51.6 \%)$. The mean age in years for the alive and dead was 62 and 70 years, respectively $(\mathrm{P}<0.05)$. Besides age, there was no statistically significant difference between those who survived compared to the ones who died in terms of demographics and coexisting comorbidities (Table 1). The most common underlying comorbidities were hypertension $(60.2 \%)$, diabetes mellitus (44.7\%), dyslipidemia (31.6\%) and congestive heart failure $(14.7 \%)$ in both the comparison groups. A significantly higher number of patients who died had a risk of mortality score categorized as extreme compared to the people who lived ( $96 \%$ vs. $70 \%, \mathrm{P}<0.05)$. ESR on admission was significantly higher among the patients who died $(\mathrm{P}<0.05)$, while CRP, $\mathrm{LDH}$, ferritin and fibrinogen were not significantly different between the two groups $(\mathrm{P}=0.36, \mathrm{P}=0.09, \mathrm{P}=0.29$, and $\mathrm{P}=0.41$, respectively). Comorbidities and the use of steroids, HCQ, TCZ or remdesivir during hospitalization were not significantly different between those who survived and those who died.

\section{Mortality}

The overall mortality was 39\%. Mortality declined significantly over time from $46 \%$ in March to $14 \%$ in August 2020 with a statistically significant difference between the months. For head-to-head comparison between individual months, we used August as a reference, and mortality of each individual month was compared against it. The adjusted odds of mortality were 
Table 1. Demographic Information, Baseline Comorbidities and Admission Laboratory Values

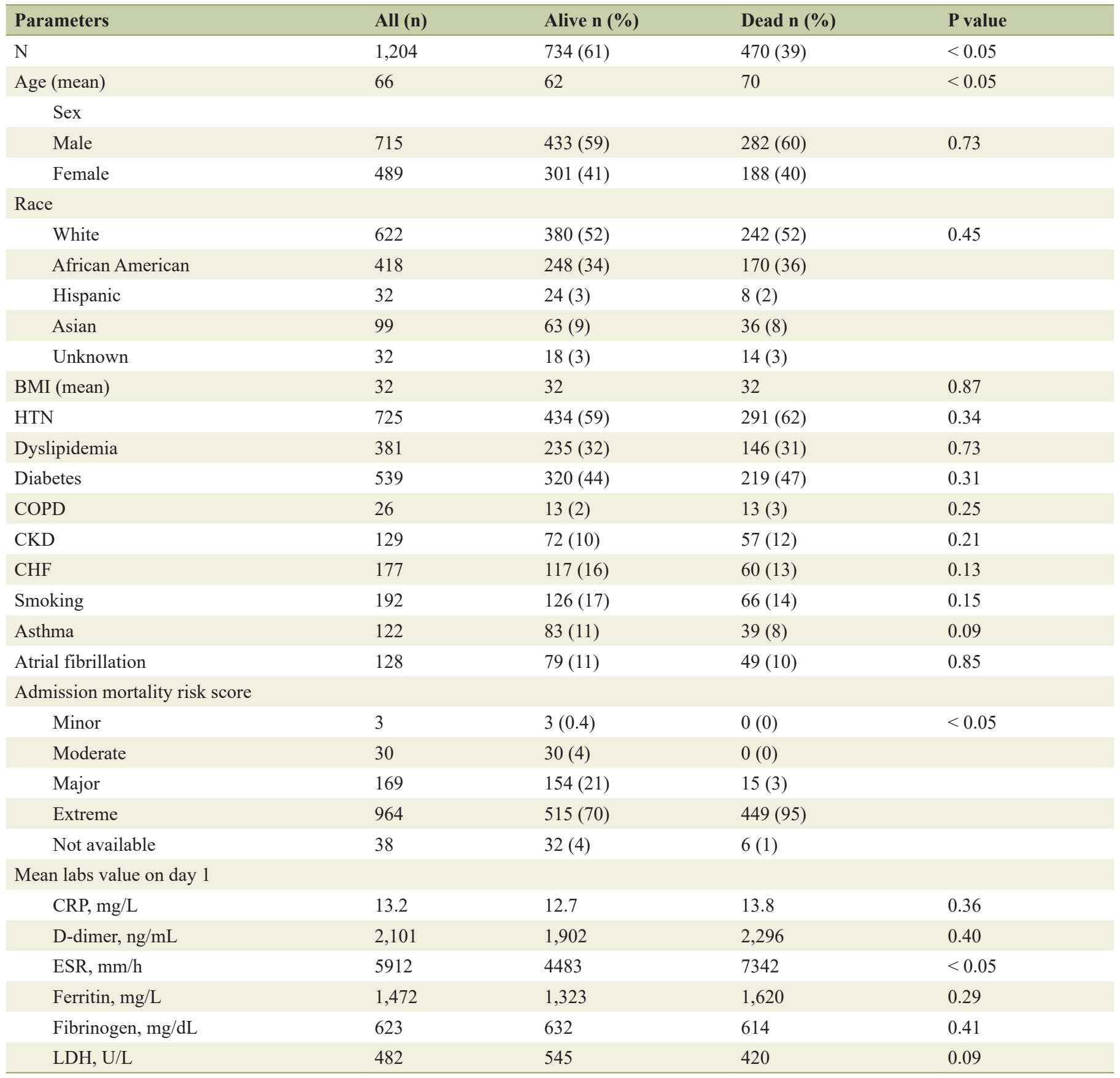

BMI: body mass index; HTN: hypertension; COPD: chronic obstructive pulmonary disease; CKD: chronic kidney disease; CHF: congestive heart failure; N: number; CRP: C-reactive protein; ESR: erythrocyte sedimentation rate; LDH: lactate dehydrogenase.

significantly lower in August as compared to March, April and May $(\mathrm{P}<0.05$, Table 2).

\section{Intervention and outcomes}

Compared to those who survived, patients who died in the ICU were more likely to require invasive mechanical ventilation
(79\% vs. $46 \%, \mathrm{P}<0.05)$, BiPAP (40\% vs. $29 \%, \mathrm{P}<0.05)$ or epoprostenol sodium ( $15 \%$ vs. $3.4 \%, \mathrm{P}<0.05$, Table 3$)$. The use of inhaled pulmonary vasodilators was relatively uncommon $(0.16 \%)$ and 27 patients $(2.24 \%)$ received ECMO. Use of HCQ was more commonly associated with the patients who died compared to the patients who survived (56\% vs. $44 \%, \mathrm{P}<$ $0.05)$, while the use of remdesivir, TCZ, steroids and duration of these medications were not significantly different between 
Table 2. Crude and Adjusted Mortality Trend by Month

\begin{tabular}{llllll}
\hline Month & Admissions (n) & Mortality (\%) & P value & AOR (95\% CI) & P value \\
\hline March & 142 & 46 & $<0.05$ & $16.81(3.59-78.78)$ & $10.25(2.58-40.79)$ \\
April & 530 & 46 & & $5.59(1.48-21.14)$ & $<0.05$ \\
May & 290 & 38 & & $3.46(.793-15.13)$ & 0.05 \\
June & 105 & 25 & & $2.73(0.64-11.58)$ & 0.18 \\
July & 88 & 23 & Reference group & \\
August & 49 & 14 & $<0.05$ & & \\
Total & 1,204 & 39 & & & \\
\hline
\end{tabular}

Factors that were adjusted for mortality trend by month included patients' age, gender, ethnicity, comorbidities (diabetes mellitus, hypertension, dyslipidemia, heart failure, smoking, chronic obstructive pulmonary disease, asthma, atrial fibrillation, chronic kidney disease, and stroke), medications (hydroxychloroquine, tocilizumab, remdesivir, steroids), admission laboratory values (CRP, ESR, D-dimer, ferritin, fibrinogen, lactate dehydrogenase) and admission risk of mortality score. AOR: adjusted odds ratio; Cl: confidence interval; CRP: C-reactive protein; ESR: erythrocyte sedimentation rate.

the two groups. Compared to the patients who survived, the peak values of ferritin, LDH, CRP and D-dimer level were significantly higher in patients who died (Supplementary Material 1, www.jocmr.org). The average hospital LOS was longer in the patients who lived compared to the patients who died at the end of hospitalization ( 18 vs. $12, \mathrm{P}<0.05)$. Majority of the patients who survived were discharged to either inpatient rehabilitation $(42 \%)$ or skilled nursing facilities $(30 \%)$.

\section{Discussion}

Our experience with critically ill COVID-19 patients showed an overall mortality lower than previous reports [5]. More importantly, we noticed a significant improvement in mortality rates over time, and this difference in mortality remained significant even after adjusting for demographics, underlying comorbidities, risk of mortality index, medications and other interventions employed during the ICU stay.

Odds of mortality were significantly higher in older patients and those with higher ESR levels on admission. Higher peak values of ferritin, LDH, CRP, D-dimer level during the hospital stay, and admission risk of mortality categorized as extreme were more likely to die. Moreover, patients who received HCQ, were on invasive mechanical ventilation, or received epoprostenol sodium during their hospital stay were more likely to die.

COVID-19 has been challenging in many aspects and its mortality has been significantly higher compared to other patients with viral pneumonia who get admitted to the ICUs [6]. This may be either due to a more deadly disease process or a sudden influx of a higher number of patients in the ICUs that were not well equipped to handle the influx. However, mortality from COVID-19 has been consistent across the globe with a trend towards a reduction in mortality with time. A metaanalysis of 24 studies comprising ICU patients revealed an overall mortality rate of $41 \%$ between the months of March to May 2020. It reduced from 50\% in March 2020 to $40 \%$ at the end of May 2020 [7]. These results are consistent with our results demonstrating a reduction in mortality over time. A ret- rospective cohort study from Wuhan, China, early in the disease course reported ICU mortality up to $90 \%$ in the patients who received mechanical ventilation during the hospital stay [8]. Our results were similar in that the overall mortality rate was $79 \%$ in the ICU patients who were intubated compared to patients who were not.

The most notable finding from our study is that mortality significantly improved from the beginning of March to August. Even after adjusting for geographical location and metaregression by month, the publications revealed that mortality had significantly decreased all around the globe over time, starting from China later followed by reports from Europe and North America [7]. Similarly, Intensive Care National Audit $\&$ Research Centre (ICNARC) reports reveal a peak at 52\% in April, and a trend towards a reduction in mortality in the following months [9]. Despite variation in admission criteria to the ICU and difference in relative proportions of patients receiving non-invasive versus invasive mechanical ventilation, with non-invasive mechanical ventilation being more prevalent in Asia than Europe and America, ICU mortality did not differ significantly across the continents. Similarly, there was a difference in the proportion of use of anti-viral drugs, immunomodulatory treatments and corticosteroids such as HCQ use more prevalent in Asia than Europe and North America, but the reduction in mortality over time has been uniform all over the world $[9,10]$.

The reasons for this reduction in mortality over time are likely multifactorial. Increased awareness with growing medical literature might have led to a reduction in mortality over time. Earlier studies were based on small patient populations and may have overestimated mortality. Another factor cited by public health officials contributing to a reduction in mortality is the shift in demographics towards the younger population making up the bulk of new infections [11]. An observational study conducted by researchers at the University of Detroit, Michigan compared the viral load of patients using the RTPCR who were hospitalized between April and June 2020. They noticed a significant reduction in the "high viral load category" from $25.5 \%$ in the first week of study to virtually zero percent in the sixth week [12]. This downward trend in initial viral load may reflect a reduction in the severity of the 
Table 3. ICU Interventions, Peak Laboratory Values and Outcomes in COVID-19 Patients

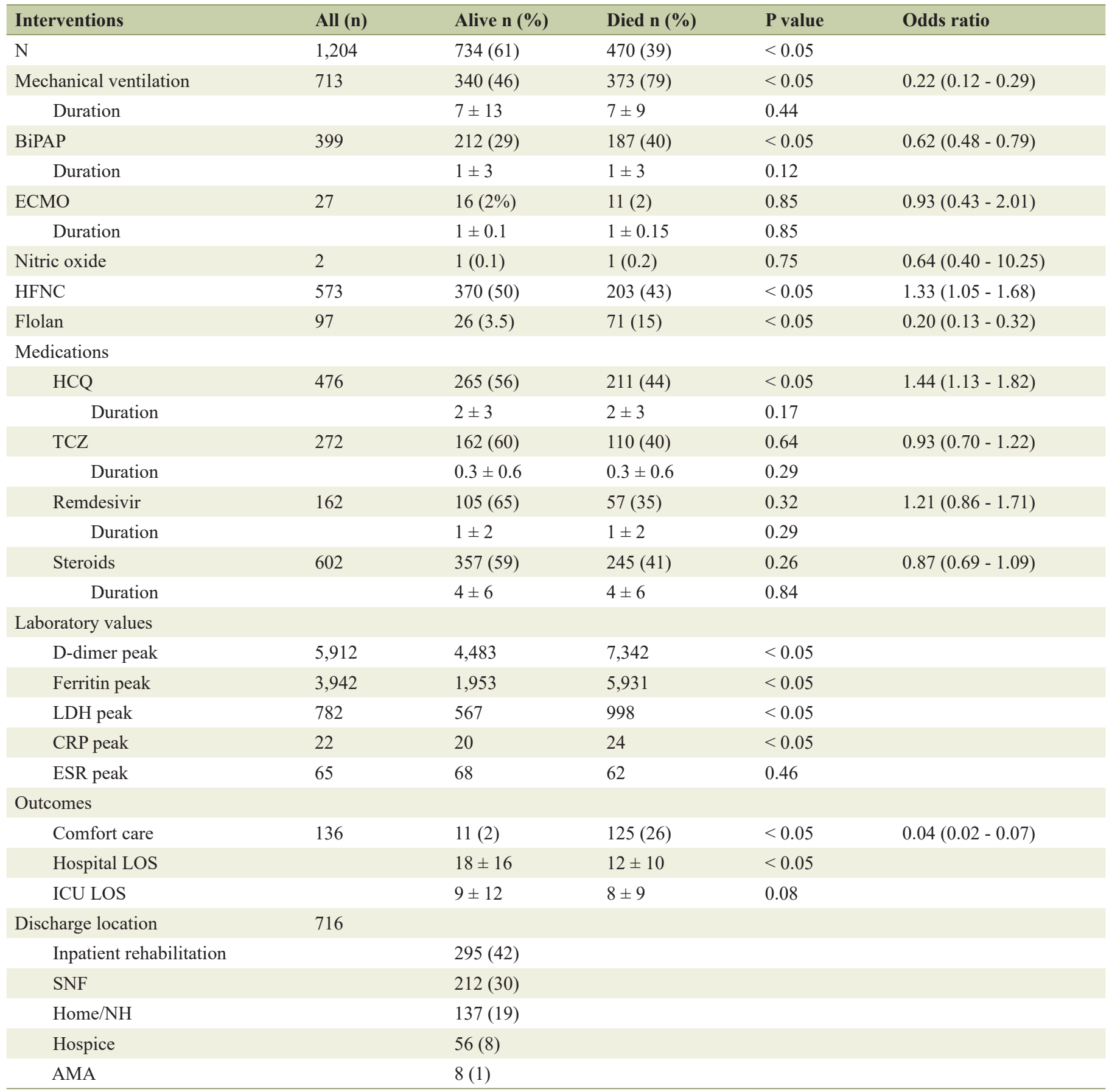

ICU: intensive care unit; BiPAP: bilevel positive airway pressure; ECMO: extracorporeal membrane oxygenation; HFNC: high-flow nasal cannula; HCQ: hydroxychloroquine; TCZ: tocilizumab; LOS: length of stay; LDH: lactate dehydrogenase; CRP: C-reactive protein; ESR: erythrocyte sedimentation rate; SNF: skilled nursing facility; $\mathrm{NH}$ : nursing home; AMA: against medical advice.

pandemic suggesting that viral load may serve as a marker to assess the progress of the pandemic. Lastly, an increase in the experience that health care providers obtained throughout the pandemic, the advancement in research through various clinical trials that have standardized various therapies despite not finding a cure and protocol improvements among institutions over time are some of the unmeasurable factors that may have contributed to this reduction in mortality over time.

Several limitations should be considered while interpreting the results of our study. A causal relationship could not be established due to the retrospective and non-randomized nature of the study. This study includes patients from 14 differ- 
ent hospitals of Jefferson Enterprise and there were unmeasurable variations in approach to patient care among all the hospitals. The impact of variations in ICU admission criteria, differences in the expertise of ICU staff, threshold for starting invasive mechanical ventilation and the initiation of several adjunctive therapies at the treating physician's discretion could not be determined. We chose laboratory values on day 1 of admission to the ICU, day 10 into ICU stay and peak values throughout ICU stay because the average duration of any therapy for COVID-19 was less than 10 days. Given the variable frequency of laboratory specimen collection, we cannot ascertain if these values represented pre and post-treatment values accurately in all cases. Lastly, our study was not designed to evaluate the contribution of underlying comorbidities to COVID-19 mortality, nor the potential adverse effects of adjunctive interventions. Many questions remain unanswered; however, by adjusting the adult patients with the confirmed disease, we believe our population is representative of the real-world cohort. Our current COVID-19 treatment practices mimic other hospital systems, and we believe these results to be generalizable.

\section{Conclusions}

Our experience with critically ill COVID-19 patients showed an overall mortality lower than previous reports. More importantly, there was a significant improvement in mortality rates over time. However, contributing factors largely remain unknown. We believe that improving our understanding of COVID-19 pathophysiology, standardizing management protocols based on continually evolving data and a decreasing viral load over time are important factors that may have improved outcomes in COVID-19 patients admitted to the ICU. Research is needed to further elucidate the factors contributing to mortality in critically ill COVID-19 patients.

\section{Supplementary Material}

Suppl 1. Comparison of labs on admission, on day 10 of hospitalization, and peak levels during hospitalization.

\section{Acknowledgments}

None to declare.

\section{Financial Disclosure}

No internal or external funding was used for this study.

\section{Conflict of Interest}

There are no conflicts of interest disclosed by any of the authors associated with this manuscript.

\section{Informed Consent}

Not applicable.

\section{Author Contributions}

Sohaib Roomi, as the main author of the article, wrote the IRB and did data analysis. Syed Omer Shah helped with the data analysis and final revision of the article. Waqas Ullah helped in data analysis. Shan Ul Abedin helped in writing the introduction. Karyn Butler helped in writing the results of the manuscript. Kelly Schiers helped in writing the methods of the study. Benjamin Kohl helped in writing the discussion. Erika Yoo provided material for comparing the results of our article with state of art literature. Matthew Vibbert did critique of the whole article. Iack Jallo did final review of the manuscript.

\section{Data Availability}

Any inquiries regarding supporting data availability of this study should be directed to the corresponding author.

\section{References}

1. Ison MG, Wolfe C, Boucher HW. Emergency use authorization of remdesivir: the need for a transparent distribution process. JAMA. 2020;323(23):2365-2366.

2. Recovery Collaborative Group, Horby P, Lim WS, Emberson JR, Mafham M, Bell JL, Linsell L, et al. Dexamethasone in hospitalized patients with COVID-19. N Engl J Med. 2021;384(8):693-704.

3. Beigel JH, Tomashek KM, Dodd LE, Mehta AK, Zingman BS, Kalil AC, Hohmann E, et al. Remdesivir for the treatment of COVID-19-final report. N Engl J Med. 383(19):1813-1826.

4. Onder G, Rezza G, Brusaferro S. Case-fatality rate and characteristics of patients dying in relation to COVID-19 in Italy. JAMA. 2020;323(18):1775-1776.

5. Auld S, Caridi-Scheible M, Blum JM, Robichaux CJ, Kraft CS, Jacob JT, Jabaley CS, et al. ICU and ventilator mortality among critically ill adults with COVID-19. medRxiv. 2020.

6. Tolksdorf K, Buda S, Schuler E, Wieler LH, Haas W. Influenza-associated pneumonia as reference to assess seriousness of coronavirus disease (COVID-19). Euro Surveill. 2020;25(11).

7. Armstrong RA, Kane AD, Cook TM. Outcomes from intensive care in patients with COVID-19: a systematic review and meta-analysis of observational studies. Anaesthesia. 2020;75(10):1340-1349.

8. Zhou F, Yu T, Du R, Fan G, Liu Y, Liu Z, Xiang J, et al. Clinical course and risk factors for mortality of adult inpatients with COVID-19 in Wuhan, China: a retrospective cohort study. Lancet. 2020;395(10229):1054-1062.

9. ICNARC report on COVID-19 in critical care 10 April 
2020. Available at: http://www.med.umich.edu/surgery/ mccen/documents/ICNARC-COVID-19-report.pdf.

10. Dunning J, Baillie JK, Cao B, Hayden FG, International Severe Acute R, Emerging Infection C. Antiviral combinations for severe influenza. Lancet Infect Dis. 2014;14(12):1259-1270.
11. Saito S, Asai Y, Matsunaga N, Hayakawa K, Terada M, Ohtsu H, Tsuzuki S, et al. First and second COVID-19 waves in Japan: A comparison of disease severity and characteristics. J Infect. 2020.

12. https://www.eurekalert.org/pub_releases/2020-09/esocuss092320.php. 